\title{
Neutrophyl - Lymphocyte Ratio (NLR) and C-Reactive Protein (CRP) Levels in Stable and Exacerbated Chronic Obstructive Pulmonary Disease (COPD) Patients in Persahabatan Hospital Jakarta
}

\author{
Astari Pranindya Sari*, Ratnawati, Wahju Aniwidyaningsih, Sita Andarini, \\ Faisal Yunus
}

Department Pulmonology and Respiratory Medicine Faculty of Medicine, Universitas Indonesia, Persahabatan Hospital, Jakarta

\begin{abstract}
Corresponding Author:
Astari Pranindya Sari| Department of Pulmonology and Respiratory Medicine, Faculty of Medicine, Universitas Indonesia, Persahabatan Hospital, Jakarta | astari.pulmo@gmail.com
\end{abstract}

Submitted: September $24^{\text {th }}, 2021$

Accepted: January $25^{\text {th }}, 2022$

Published: February $28^{\text {th }}, 2022$

Respir Sci. 2022; 2(2): 78-91

https://doi.org/10.36497/respirsci.v2i2.38

\begin{abstract}
Background: Although COPD has been believed to be characterized by respiratory disease, there are currently limited study conducted to evaluate inflammation markers and exacerbation rate in COPD by noninvasive methods. We did a prospective cohort study to observe the alteration of Neutrophyl-Lymphocyte Ratio (NLR) and C-reactive protein (CRP) in COPD patients to find any possible correlation with COPD exacerbation status.

Method: From July to December 2018, a prospective cohort study was performed with blood and spirometry test on 31 COPD patients during exacerbation (AE-COPD) and stable. The mean of NLR and CRP were compared and analyzed.
\end{abstract}

Results: Both NLR and CRP decreased during stable condition (from $7.95 \pm 6.8$ to $4.6 \pm 5.5$ and $43.4 \pm 71$ to $12.2 \pm 18.5$ ) with $P<0.01$ respectively. We also found a significant inverse correlation between NLR and $F E V_{1} / F V C$ in $A E-C O P D$ and CRP showed an inverse correlation only with $\mathrm{FEV}_{1}$ in $\mathrm{AE}-\mathrm{COPD}$. Another interesting finding was a subject with very high CRP whose value remained above the nomal limit during stable, and died within 2 months after exacerbation.

Conclusion: NLR and CRP levels in COPD patients increased during exacerbation, which may reflect lung function and exacerbation status.

Keywords: neutrophil, lymphocyte, COPD, NLR, CRP

\section{INTRODUCTION}

Several previous studies have concluded that neutrophils are the dominant cells exacerbating COPD. ${ }^{1-4}$ In addition to airway conditions, COPD has the characteristics of a systemic inflammatory disease. ${ }^{5}$ Research in recent years has proven the value of the neutrophil-lymphocyte ratio (NLR) in blood samples as a potential marker of systemic inflammation. ${ }^{6}$ In 2014, Günay et al. concluded NLR could be used as an inflammatory marker to assess the degree 
of inflammation in COPD. This NLR test is characterized as fast, inexpensive, and easy. ${ }^{7}$

Research on NLR showed an association between NLR and airflow limitation, disease severity, exacerbations, hospitalizations, and mortality in COPD..$^{8-11}$ Considering COPD has systemic inflammation characteristics, this study will also assess C-reactive protein (CRP), which was stated to be increased in patients with acute exacerbated COPD (AE-COPD) compared to stable conditions. ${ }^{5,9}$ Research that found evidence that the NLR in AECOPD was higher than stable COPD was mostly designed as cross-sectional or retrospective. ${ }^{9-11}$ In Indonesia, there is no COPD research focused on NLR or CRP yet.

\section{METHOD}

This study is a descriptive-analytic with prospective cohort design. It was carried out at the emergency department and outpatient clinic of Persahabatan Hospital Jakarta from July 2018 to December 2018. A total of 31 AE-COPD patients who met the inclusion criteria were subjected to spirometry and blood tests and then followed up for one month after the exacerbation to be re-examined when their condition was stable.

No patients complained of shortness of breath since childhood or experienced shortness of breath before smoking. Patients with possible Post-Tuberculosis Obstructive Syndrome (SOPT) were excluded through history taking and chest $\mathrm{X}$-rays during the inclusion process.
All data obtained were analyzed using SPSS 20.0 for windows. Saphiro Wilk normality test was done to determine the data distribution with fewer than 50 subjects followed by a comparison test of two mean paired data sets with Wilcoxon and paired t-test, respectively to the normality test results. Spearman or Pearson was used to find a correlation.

\section{RESULT}

This study recruited 39 AE-COPD patients who met inclusion criteria. Eight patients did not participate until the analysis step due to drop outs: 2 patients dealt with administration regarding the referral process, 4 patients lost contact and did not show up on the specified date, and 2 patients withdrew themselves. Therefore, 31 patients participated in this study until the final stage.

The average age was $62 \pm 1.5$ years, with Body Mass Index (BMI) of 21.8. Airway obstruction severity according to the GOLD criteria (defined by percentage of $\mathrm{FEV}_{1}$ /predicted $\mathrm{FEV}_{1}$ ) shows that most of the patients in this study were GOLD II criteria ( 25 patients - $80.6 \%$ ). In GOLD III group, there were 5 patients (16.3\%), and only one patient included in GOLD I criteria. There were no patients who met GOLD IV.

In both exacerbation and stable conditions, the patient filled out a form, resulting in two CAT scores being obtained for each patient. The average CAT score in AE-COPD was $15.4 \pm 3.8$, while stable was $12.2 \pm 2.4$. Only two patients (6.45\%) 
showed similar CAT scores while 93.5\% experienced a decrease in CAT scores during stable $(P<0.01)$. There were no patients with higher CAT scores during AECOPD.

Table 1. Characteristics of the research patients

\begin{tabular}{lc}
\multicolumn{1}{c}{ Characteristics } & $\begin{array}{c}\text { Subject } \\
\mathbf{N}=\mathbf{3 1}\end{array}$ \\
\hline Age (years) & $62 \pm 1.5$ \\
Body Mass Index (BMI) & 21.8 \\
Smoking status & \\
Active & 2 \\
Ex-smoker & 29 \\
Brinkmann Indeks Index & \\
Mild & 0 \\
Medium & 5 \\
Heavy & 26 \\
Pre-bronchodilator spirometry & \\
(obtained during stable) & \\
FEV ${ }_{1}$ & $1387.2 \pm 40$ \\
FVC & $2046.4 \pm 52$ \\
FEV ${ }_{1} /$ FVC & $62.4 \pm 0.7$ \\
Post-bronchodilator spirometry & \\
(obtained during stable) & \\
FEV & \\
FVC & $1532.4 \pm 41.5$ \\
FEV ${ }_{1} /$ FVC & $2354 \pm 70$ \\
GOLD* Criteria & $65.2 \pm 0.6$ \\
I & \\
II & \\
III & \\
IV & 25 \\
\hline Note: *GOLD: Global Initiative for Obstructive Lung \\
Disease
\end{tabular}

The results obtained showed that the $\mathrm{FEV}_{1}$ and FVC values were lower in AECOPD. The average $\mathrm{FEV}_{1}$ was $666.29 \pm 52$ (AE-COPD) and 1350 \pm 41 (stable). Data with $\mathrm{n}<50$ using the Saphiro-Wilk showed $P>0.05$ (normal distribution), resulting in paired t-test analysis was used for comparison of two paired means. Based on the study, the results were statistically significant with $P<0.01$. Meanwhile, the FVC also showed a statistically significant difference, $1258.38 \pm 55$ in AE-COPD and $1978 \pm 53$ at stable $(P<0.01)$.

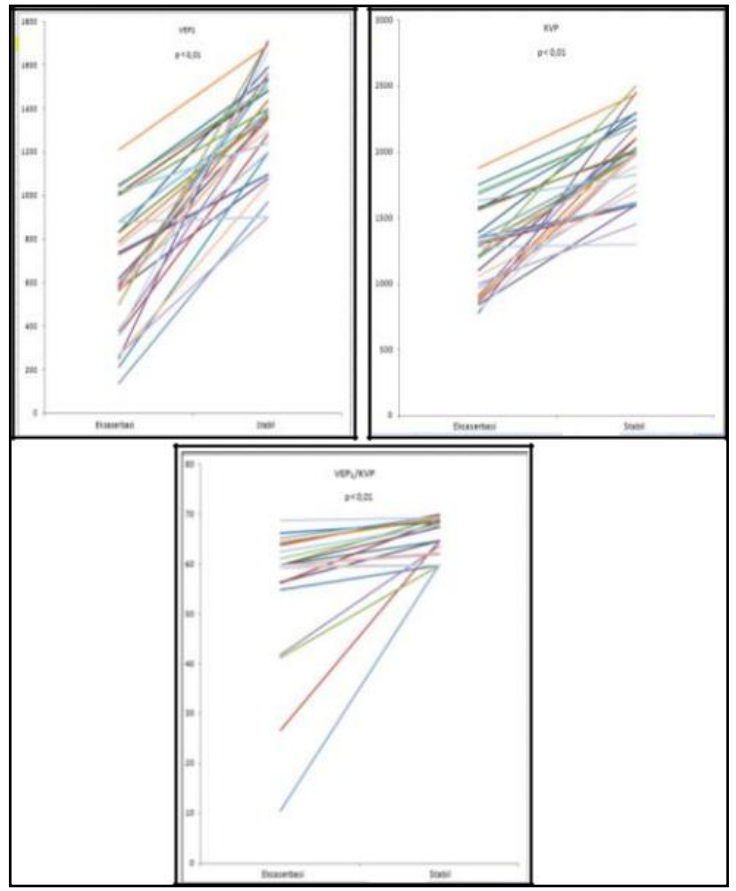

Graphic 1. Changes in the patient's pulmonary function in exacerbation and stable conditions

Airway obstruction characterized by $\mathrm{FEV}_{1} / \mathrm{FVC}$ also changed. In AE-COPD, $\mathrm{FEV}_{1} / \mathrm{FVC}$ was lower (56.8 \pm 2 vs $64 \pm 0.8$ ). The $\mathrm{FEV}_{1} / \mathrm{FVC}$ data distribution was not normal, Wilcoxon test was used to compare the two paired mean data. The analysis showed $P<0.01$. Changes in FVC and $\mathrm{FEV}_{1}$ values in these two different conditions are shown in Graphic 1.

NLR

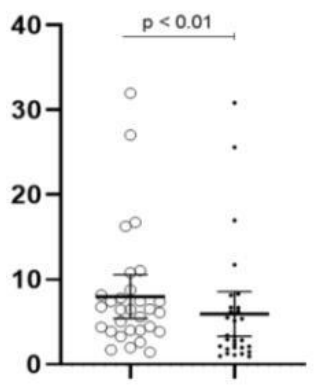

Graphic 2. Average NLR and CRP values in exacerbation and stable conditions

In $A E-C O P D$, the NLR average was $7.95 \pm 6.8$, while at stable $4.6 \pm 5.5$. A total of 28 patients showed a decrease in NLR 
during stable conditions. Three patients showed a higher NLR in a stable condition. Meanwhile, CRP levels in 10 patients showed higher levels at stable condition than exacerbation. The CRP average in AECOPD was $43.4 \pm 71$, while the stable condition was $12.2 \pm 18.5$. The difference was statistically significant based on the Wilcoxon test $(P<0.01)$. Graphic 2 shows the average difference between the two variables.

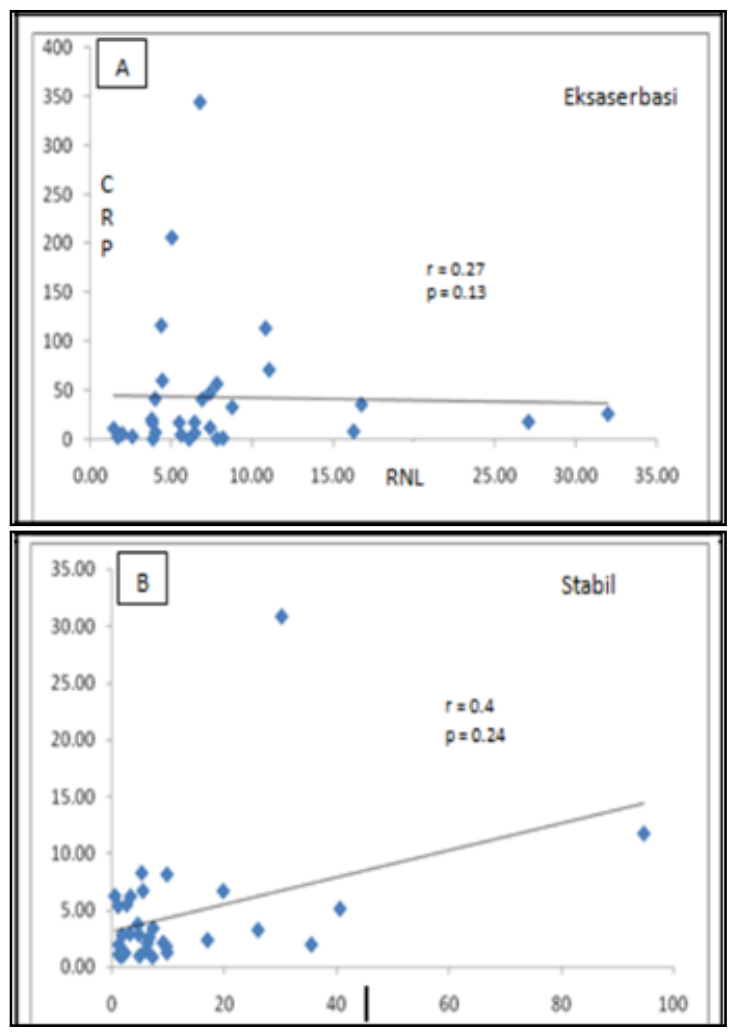

Graphic 3. A) Correlation between NLR and CRP values at exacerbation. B) Correlation between NLR and CRP at stable condition

The results of this study indicate that there is no correlation between NLR and CRP. Correlation data analysis was carried out with the Spearman correlation test for quantitative data with a non-normal distribution. In addition, the NLR and CRP values shown in Graphic 3A were analyzed in an exacerbation condition.

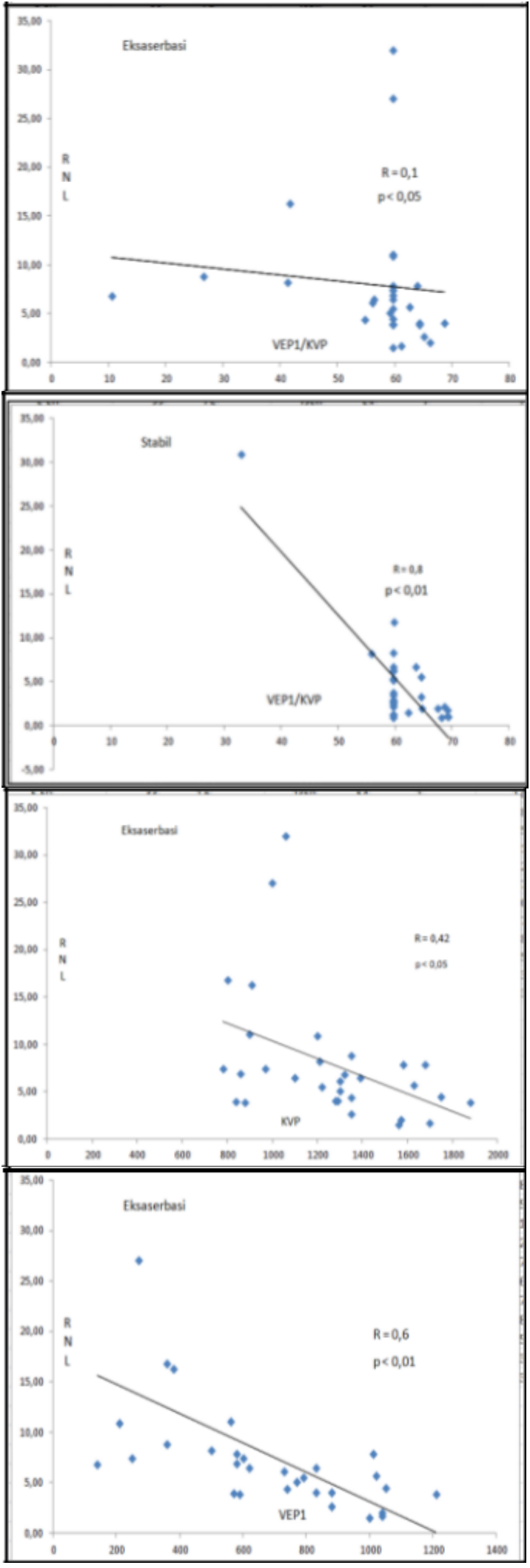

Graphic 4. Correlation of NLR with FVC and FEV1

The correlation between NLR and FVC and $\mathrm{FEV}_{1}$ was analyzed using Spearman and found to have an inverse correlation 
(negative). As in NLR with CRP, data processing of NLR and spirometry results were also based on exacerbation status. The correlation coefficient between NLR and FVC in AE-COPD and stable was $r=$ -0.42 and $r=-0.17$, respectively.

NLR also showed a negative correlation with $\mathrm{FEV}_{1}$. In AE-COPD, the value of the correlation coefficient $r=-0.6$ while in stable conditions is -0.54 . Airway obstruction, as indicated by the $\mathrm{FEV}_{1} / \mathrm{FVC}$, also showed a negative correlation. The correlation coefficient value of NLR with $\mathrm{FEV}_{1} / \mathrm{FVC}$ at exacerbation is $r=-0.1$ with $P<0.05$. In stable conditions, Spearman's analysis showed a significant correlation $(r=-0.8 ; P<0.01)$.

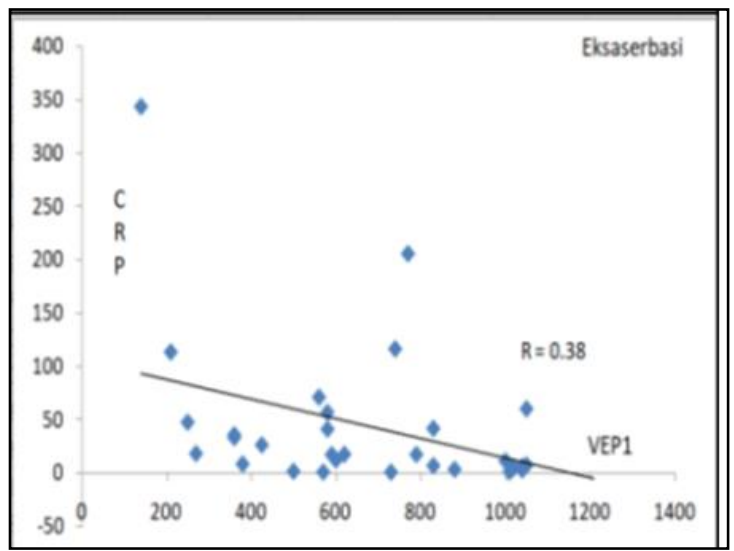

Graph 5. Correlation between CRP value with FVC and FEV1

CRP also showed a negative correlation. In AE-COPD, there was no significant correlation between CRP and FVC with $r=-0.04$, while in the stable condition, it was -0.26 . The correlation coefficient of CRP with $\mathrm{FEV}_{1}$ in the exacAECOPD and stable group was $r=-0.38$ and $r=-0.27$, respectively. There is no correlation between these two variables with $\mathrm{FEV}_{1} / \mathrm{FVC}(r=-0.17)$ in the exacerbation condition $(P=0.07)$ and $r=$ $-0.24(P=0.3)$ in the stable condition.

The Spearman correlation test showed no correlation between CRP and CAT scores in both exacerbating and stable conditions. The correlation coefficient obtained was $r=0.17 \quad(P=0.37)$ at exacerbation and $r=0.32$ when stable with $\mathrm{P}=0.08$. Similarly, NLR did not show a correlation with CAT.

There is no correlation between the CAT score and $\mathrm{FEV}_{1} / \mathrm{FVC}$ in both exacerbated and stable condition. This study tends to find that the CAT score can change based on the exacerbation status in the same patient. In stable conditions, the CAT value was lower but not correlated with $\mathrm{FEV}_{1} / \mathrm{FVC}$. The correlation coefficient values of CAT with $\mathrm{FEV}_{1} / \mathrm{FVC}$ in exacerbation and stable conditions were $r=-0.28(P=0.94)$ and $r=-0.16(P=0.37)$.

Based on the Spearman correlation analysis, the NLR and CRP in AE-COPD did not correlate with exacerbation incidence within 1 month after the last exacerbation. Seven patients (21\%) experienced one relapse within one month after the previous exacerbation. The mean NLR of exacerbations of this one-time relapse was 5.7. As for CRP, the average level in patients who experience one recurrence in one month is 77.5.

Two patients died after data collection. The patient died within one month of completing data collection. One patient who died recorded his NLR and CRP in AE-COPD 3.8 and 17, while when stable, they decreased to 2.1 and 6.3. The other patients showed NLR and CRP values of 6.8 
and 343.9 (AE-COPD) while those stable were 11.7 and 94.7 , respectively. The first patient died with a history of no improvement in high $\mathrm{pCO}_{2}$ on blood gas analysis. Meanwhile, the other patients had no known history of the cause of death. The increase in NLR when stable was shown by patients who died above and other patients, from 2.61 at exacerbation to 6.2 when stable. In contrast to patients who died, the CRP value in these patients decreased from 3.1 to 0.5 .

This patient was known to have experienced 1 exacerbation in one month after examining a stable condition. There were three patients with higher CRP in stable, experiencing exacerbations within one month after the regular period, with one case requiring treatment in the intensive care unit for indications of type 2 respiratory failure.

The other seven patients had no exacerbations within one month. The mean CRP of these patients was 3.3, while the three patients who had exacerbations returned, showing a mean CRP of 14.3 at the time of the previous exacerbation. One patient showed a high CRP value of 205.8 at the time of exacerbation. This patient had no recurrence of exacerbations within one month of the worsening. The patient showed a drastic decrease in CRP, which was down to 9.8 in a stable condition. Patients with higher CRP values, when stable, who experienced a recurrence of exacerbations within one month were not accompanied by an increase in NLR while stable.
The GOLD criteria were made based on the patient's spirometry results while in stable condition. Therefore, the difference in mean shows an increase as the GOLD criteria increase. However, as previously explained, the number of patients in each group of GOLD criteria was not balanced. There were no patients on GOLD IV, and there was only one patient on GOLD I. Statistically, the mean difference that was significant was only indicated by the CRP value. The patient is in stable condition.

\section{DISCUSSION}

Former smokers dominated patients in this study with severe Brinkmann Index scores. This background is a risk factors for COPD. Some patients met the criteria for COPD GOLD II.

CAT scores decreased when conditions were stable compared with exacerbations in the same individual. It is known that the value obtained from the CAT score is an important part of COPD patients' management. A Japanese study conducted by Suetomo in 2014 that focused on studying whether CAT could be used to predict exacerbations in COPD patients showed that CAT could predict exacerbations. However, this study did not find evidence that CAT can indicate the need for hospitalization in COPD patients because not all COPD patients who experience exacerbations are hospitalized. It is said that the intention of the CAT to facilitate communication between doctors and patients regarding 
the effects of a disease that occurs in patients with COPD. ${ }^{12}$

Another study conducted by Ghobadi in 2011 concluded that the CAT score is closely related to the severity of airway obstruction. ${ }^{13}$ However, the results mean as in the study was not found in this study. There are two elements in the CAT assessment, namely respiratory and nonrespiratory symptoms. ${ }^{14}$ In this study, the patients' improvements in the CAT score varied.

This study showed that the NLR of AE-COPD patients was significantly higher. Considered the GOLD criteria, the NLR value in stable condition shows an average difference. The NLR mean of GOLD II and III in AE-COPD was 6.24 and 14.74 , respectively. Meanwhile, in stable patients 3.7 and 8.7. In GOLD I group, there was only one patient and this patient had an NLR of 16.75 (AE-COPD) and 5.14 when stable.

A previous study conducted in Japan (2011) by Furutate et al. found an increase in NLR value as the degree of airway obstruction worsened. In this study, the increase could be in line with the rise in GOLD criteria. The result of this study showed a similar trend, but the patient number of each subject was not balanced. Considering this fact, the suitability of the increase in NLR with the degree of airway obstruction could not be further assessed. Nevertheless, the analysis of the difference in mean NLR between the GOLD groups showed a statistically significant difference
$(P<0.05)$ in both exacerbation and stable conditions. $^{9}$

It is known based on the GOLD guidelines that $\mathrm{FEV}_{1} / \mathrm{FVC}$ below $70 \%$ post-bronchodilator is a criterion for establishing a COPD diagnosis. This value also describes the severity of airway obstruction. In contrast to the explanation in the previous paragraph regarding NLR and the GOLD criteria, the correlation test between NLR and $\mathrm{FEV}_{1} / \mathrm{FVC}$ showed a significant correlation. From Graphic 4, NLR is negatively correlated with $\mathrm{FEV}_{1} / \mathrm{FVC}$ in both AE-COPD and stable. These results are consistent with previous studies, which consistently found that high NLR was associated with decreased lung function. In contrast to the analysis of prior studies, this study distinguishes between exacerbation and stable analysis.

Research by Xiong et al. in 2017 also found a significant correlation between NLR and COPD severity. Xiong analyzed prognostic factors and concluded that NLR was correlated with mortality. In contrast to the objectives of this study, this study did not aim to find a correlation between NLR and mortality. ${ }^{5}$ However, two patients died after the study was completed. One of the patients who died showed increased NLR when they were stable. It can be argued that this case supports Xiong's finding that NLR may be a predictor of mortality in COPD patients.

In 2015, Lee et al. obtained results showing that the NLR value of 2.8 is the limit value for predicting the risk of 
hospitalization caused by exacerbations in COPD patients. $^{6}$ These results are consistent with this study, which obtained an average NLR of 7.95 in AE-COPD requiring hospitalization. The theory states that COPD is a persistent systemic inflammatory disease. This study did not involve healthy patients as controls, but there was a clear difference in the NLR mean during stable versus exacerbation conditions in the same individual.

Several mechanisms may explain the increased systemic inflammation in COPD. The first possibility is the spillover of inflammatory mediators from the pulmonary compartment. In addition, another possibility is in response to the presence of tissue hypoxia. It is known that COPD patients experience tissue hypoxia on average due to insufficient respiratory conditions. The third possibility is caused by the immune system's response to bacterial infections that cause COPD exacerbations. For example, for gram-negative bacteria, the lipopolysaccharide (LPS) component of these bacteria will be recognized by TLR4 on the surface of innate immune cells as antigens. ${ }^{7}$

Furthermore, proinflammatory pathway activation will produce cytokines and chemokines to recruit more immune cells, including neutrophils and lymphocytes. Neutrophils are increased in COPD patients who experience exacerbations due to bacterial infection, but this does not mean that bacterial infection is the only cause of increased neutrophil levels. ${ }^{7}$
The deficiency of alpha-1 antitrypsin is a risk factor for developing COPD in a person. Still, because this case is considered as rare, especially in Asian races, neutrophils are the cause of an imbalance between proteases and antiproteases in the lungs. Neutrophils recruited to the walls of the airways and lungs as an innate immune cell reaction to infection will produce oxygen radicals and proteolytic enzymes such as proteases, causing tissue damage. This pathophysiology describes the emphysematous type of COPD. Likewise, in chronic bronchitis, several studies that focus on the analysis of airway smooth muscle have shown a relationship between the degree of neutrophil infiltration and the severity of airway obstruction. ${ }^{7,15}$

Discussion of lymphocytes, which are components of NLR, has been shown by several studies that found infectious pathogens were the background for the increase in lymphocytes in patients. Most of the $\mathrm{CD}^{+} \mathrm{T}$ lymphocytes in patients with COPD are versions of memory $T$ lymphocytes. It means that in COPD patients, there has been the introduction of infecting pathogenic bacteria that produce $\mathrm{CD}^{+} \mathrm{T}$ lymphocytes in $\mathrm{T}$ cell differentiation and then produce memory $\mathrm{T}$ cells as one of the differentiation process results. Memory $\mathrm{CD}^{+} \quad \mathrm{T}$ lymphocytes will then respond very quickly to infecting bacteria in subsequent infections, and this response is the background for exacerbations and other immune components. ${ }^{15}$ 
High lymphocyte counts in stable COPD patients may have two meanings: as a result of previous exacerbations and as an indication of the immune system "understanding" and "responding" that a person's respiratory system needs more protection. ${ }^{15}$ Low lymphocyte count or lymphopenia in some cases of infection such as sepsis, bacteremia, and some chronic diseases such as cardiovascular disease and cancer are associated with a poor prognosis. In some COPD patients, lymphopenia is associated with advanced age and poor nutritional status. ${ }^{8}$

There is a postulate from Xiong et al.'s study that NLR, which integrates neutrophils and lymphocytes, shows more association with COPD than neutrophils or lymphocytes alone. ${ }^{5}$ A high NLR value, which means neutrophilia and lymphopenia together, indicates inflammation and a decreased immunity, as well as non-optimal health conditions. This is following COPD's essence, a systemic inflammatory and immunocompromised condition.

Three patients in this study showed a higher NLR in stable condition compared to exacerbation. In addition, the patient showed a stable condition of lymphopenia, resulting in a higher neutrophil to lymphocyte ratio value. This is related to age and poor nutritional status. The three patients were aged 75 , 81 , and 66 , respectively with a body mass index of 16,17 and 21 . Some of these results show agreement with the theory that lymphopenia in COPD can occur in geriatrics and poor nutritional status. For example, when the condition was stable, one of the five patients with high NLR values was 81 years old with a BMI of 17 .

This study also observed the CRP levels of COPD patients. The average CRP in AE-COPD was much higher than stable. High CRP levels are inversely correlated with airway obstruction. This study found that $F E V_{1}$ is associated with NLR, but no correlation was found between NLR and CRP. Related to the basic theory of neutrophil recruitment by proinflammatory cytokines, this result is not representative.

The ratio of neutrophils to lymphocytes consists of two important components in inflammation: neutrophils and lymphocytes. High levels of NLR can indicate the number of neutrophils in the blood, which is a continuation response of Interleukin (IL)-8 produced by innate immune cells. Interleukin (IL)- 8 is a cytokine and chemokine that is produced together with other innate proinflammatory cytokines such as IL-1, IL2, IL-6, IL-12, and TNF-alpha. Neutrophils have a direct relationship with IL-8, as well as IL-6. Furthermore, hepatocytes produce CRP in response to IL-6, which is captured by hepatocyte receptors. ${ }^{11}$ Theoretically, this indicates that the CRP value should be in line with the NLR, but this was not found to be the case in this study. Although NLR and CRP together showed a decrease in levels when the condition was stable, some patients experienced an increase in CRP in stable condition, not including those who also experienced an increase in NLR 
when stable. The analysis results that do not show a correlation can be caused by this explanation.

Another factor is that some patients who showed high NLR have lymphopenia, therefore the NLR count results are high. This could be due to the low number of proinflammatory cytokines produced by innate immune cells, including IL-6 cytokines, resulting in NLR and CRP values obtained did not show a correlation. In patients who show a high NLR with lymphopenia condition, there are not many neutrophils, so there are few proteases. As a result, tissue damage can be a stimulus for the production of proinflammatory cytokines. In this case, the not-so-much IL-6 is followed by low CRP, which causes a weak correlation between NLR and CRP in the overall analysis.

The absence of CRP and NLR correlations obtained from this study, although not by previous studies, actually shows the line with theory regarding the pathophysiology of CRP production by the liver. Infection can be caused by a variety of infectious agents other than bacteria. An example is a virus. The incidence of viral infections in the respiratory tract that can cause acute exacerbations in patients with COPD is quite high. ${ }^{16}$

In this regard, the effect of CRP produced by the liver may be different from that of produced due to bacterial infection. The theory shows that virus infection will result in a low CRP value, while a bacterial infection will cause a significant increase in CRP.
The pathways of immunological responses to bacteria and viruses differ from one another. As mentioned above, macrophages will respond first by phagocytosis and producing proinflammatory cytokines in response to bacterial infections. Among several proinflammatory cytokines, there is IL- 6 which will be captured by receptors on hepatocytes, followed by the production of CRP by the liver. The process is different in conditions of viral infection. Interferon (IFN) is a proinflammatory cytokine predominantly produced in response to viral infection. This interferon-dominated immunological response could be the background for the Iow CRP produced by hepatocytes even though it is still produced as an inflammatory response.

This background may be the reason for the lack of a correlation between NLR and CRP in this study. The causes of exacerbations were not examined further, resulting in a not-known cause of exacerbation (by bacteria or viruses). A significant increase in CRP in some patients might be caused by a bacterial infection, while a virus infection can cause a small increase in CRP. In the previous discussion, it was mentioned that there is a possible correlation between high NLR counts due to lymphopenia and age and nutritional status. ${ }^{8}$

In this study, it was also found that patients who showed high CRP values in stable conditions had a BMI below normal with a mean of 18.4. This result is lower than patients who showed decreased CRP 
in a stable condition: 21 . In addition, CRP levels showed an inverse correlation with $\mathrm{FEV}_{1}$, which is an inverse correlation. These results indicate that the consequences of COPD are not only directed toward towards the respiratory system but also extrapulmonary and systemic.

This result is interesting because COPD is a chronic inflammatory condition, while CRP is an acute-phase protein produced by hepatocytes in response to inflammation or tissue damage. The study conducted by Gan et al. is the first to derive significant value from high levels of CRP in COPD patients. The study showed that the results of CRP increased in active smokers, patients with decreased lung function, stable COPD patients, and even high levels of CRP were predictors of mortality related to cardiovascular disease. ${ }^{16}$

Other studies have shown that patients with COPD have higher CRP levels, which is not associated with cardiovascular disease. However, it was also found that CRP can predict mortality in COPD patients, and CRP levels can decrease with routine exercise. ${ }^{17-19}$

The study by Furutate et al. found a strong correlation between CRP and NLR in COPD patients, but the study did not mention the possibility of CRP as a predictor of mortality in COPD. ${ }^{9}$ Meanwhile, Yousef said that NLR was more likely to represent exacerbations in COPD patients than other inflammatory markers such as CRP. ${ }^{20}$ In this study, there are several noteworthy findings regarding the usefulness of both NLR and CRP. Two patients in this study died, and one showed very high CRP levels (>300) at the time of exacerbation.

In this case, although CRP levels decreased when stable within one month later, the levels were still far above the normal value. Simultaneously, these patients also showed an increased NLR when stable compared to exacerbations. Other patients who died also showed CRP levels decreased when stable but remained above the maximum reference value. In contrast to the previous death case, this one was not accompanied by an increase in NLR. Meanwhile, the correlation test from this study did not show a correlation between CRP and the frequency of exacerbations within one month.

Several CRP values which showed an increase when stable compared to exacerbations showed a possible correlation with exacerbation recurrence within one month. Interestingly, there are some patients with an increase in CRP when stable, but the CRP value was not too high during the previous exacerbation, did not experience a recurrence of exacerbation within one month. Likewise, the finding that an increase in NLR does not accompany an increase in CRP does not show an association with death in COPD patients. It is important data and may be used as the basis for further research to explore the possibility of NLR and CRP as predictors of mortality in COPD patients at Persahabatan Hospital. 
This study has limitations, including not including healthy people without COPD as the control group. In addition, in this study, the number of samples in each GOLD group was not balanced. This is the reason for the inconsistent increase in NLR and CRP and the increase in the GOLD criteria. The cause of the exacerbation in this study was also not examined further, whether it was a bacterial or viral infection. It affects the various increases in CRP. Another limitation of this study was that not all patients could prove the use of inhaled drugs within the framework of COPD treatment correctly because some patients did not take their medication with them at the time of the examination. It may be a confounding variable in this study.

\section{CONCLUSION}

NLR values in COPD patients were higher in exacerbation conditions than in stable conditions. The NLR value showed an inverse correlation with $\mathrm{FEV}_{1}, \mathrm{FVC}$, and $\mathrm{FEV}_{1} / \mathrm{FVC}$ only in exacerbation conditions in COPD patients. There is no correlation between NLR scores and CAT scores in COPD patients. There is no correlation between NLR and CRP values.

Further research is needed to include healthy people without COPD, smokers and non-smokers to determine the NLR and CRP values. In addition, it is necessary to conduct a study with a balanced number of patients on each of the GOLD criteria. Another thing that adds to further research is the classification of causes of exacerbations, such as bacterial infections, viruses or other causes. The high CRP value in this study indicates a possible correlation with exacerbations and mortality, but this is not included in the purpose of this study. But this evidence can be the basis for further research to focus more on CRP as a predictor of recurrence and mortality in COPD.

\section{REFERENCES}

1. Siva R, Green RH, Brightling $C E$, et al. Eosinophilic airway inflammation and exacerbations of COPD: a randomised controlled trial. Eur Respir J. 2007;29(5):906-913.

2. Bafadhel M, Davies L, Calverley PMA, Aaron SD, Brightling CE, Pavord ID. Blood eosinophil guided prednisolone therapy for exacerbations of COPD: a further analysis. Eur Respir $J$. 2014;44(3):789-791.

3. Rutgers SR, Postma DS, Ten Hacken $\mathrm{NHT}$, et al. Ongoing airway inflammation in patients with COPD who do not currently smoke. Thorax. 2000;55(1):12-18.

4. Drost EM, Skwarski KM, Sauleda J, et al. Oxidative stress and airway inflammation in severe exacerbations of COPD. Thorax. 2005;60(4):293300.

5. Xiong $\mathrm{W}, \mathrm{Xu} \mathrm{M}$, Zhao $\mathrm{Y}, \mathrm{Wu} \mathrm{X}$, Pudasaini B, Liu JM. Can we predict the prognosis of COPD with a routine blood test? Int $J$ Chron Obstruct Pulmon Dis. 2017;12:615-625.

6. Lee $\mathrm{SJ}$, Lee $H R$, Lee $T W$, et al. Usefulness of neutrophil to 
lymphocyte ratio in patients with chronic obstructive pulmonary disease: a prospective observational study. Korean J Intern Med. 2016;31(5):891.

7. Günay E, Sarınç Ulaşlı S, Akar O, et al. Neutrophil-to-lymphocyte ratio in chronic obstructive pulmonary disease: a retrospective study. Inflammation. 2014;37(2):374-380.

8. Sørensen AK, Holmgaard DB, Mygind LH, Johansen J, Pedersen C. Neutrophil-to-lymphocyte ratio, calprotectin and $\mathrm{YKL}-40$ in patients with chronic obstructive pulmonary disease: correlations and 5-year mortality - a cohort study. J Inflamm (Lond). 2015;12(1).

9. Furutate $R$, Ishii $T$, Motegi $T$, et al. The Neutrophil to Lymphocyte Ratio Is Related to Disease Severity and Exacerbation in Patients with Chronic Obstructive Pulmonary Disease. Intern Med. 2016;55(3):223-229.

10. Lee $H$, Um SJ, Kim YS, et al. Association of the Neutrophil-toLymphocyte Ratio with Lung Function and Exacerbations in Patients with Chronic Obstructive Pulmonary Disease. PLoS One. 2016;11(6).

11. Kim YJ, Lim B, Kyung SY, Park J, Jeong $\mathrm{SH}$. Comorbidity and Inflammatory Markers May Contribute to Predict Mortality of High-Risk Patients With Chronic Obstructive Pulmonary Disease Exacerbation. $J$ Clin Med Res. 2016;8(7):531.

12. Suetomo M, Kawayama T, Kinoshita T, et al. COPD assessment tests scores are associated with exacerbated chronic obstructive pulmonary disease in Japanese patients. Respir Investig. 2014;52(5):288-295.

13. Ghobadi H, Ahari SS, Kameli A, Lari SM. The Relationship between COPD Assessment Test (CAT) Scores and Severity of Airflow Obstruction in Stable COPD Patients . Tanaffos. 2012;11(2):22-26.

14. Houben-Wilke $S$, Janssen DJA, Franssen FME, Vanfleteren LEGW, Wouters EFM, Spruit MA. Contribution of individual COPD assessment test (CAT) items to CAT total score and effects of pulmonary rehabilitation on CAT scores. Health Qual Life Outcomes. 2018;16(1):1-8.

15. O'Donnell R, Breen D, Wilson $S$, Djukanovic R. Inflammatory cells in the airways in COPD. Thorax. 2006;61(5):448-454.

16. Gan WQ, Man SFP, Senthilselvan A, Sin DD. Association between chronic obstructive pulmonary disease and systemic inflammation: a systematic review and a meta-analysis. Thorax. 2004;59(7):574-580.

17. Pinto-Plata VM, Müllerova $\mathrm{H}$, Toso JF, et al. C-reactive protein in patients with COPD, control smokers and nonsmokers. Thorax. 2006;61(1):23-28.

18. Cano NJM, Pichard C, Roth $\mathrm{H}$, et al. Creactive protein and body mass index predict outcome in end-stage respiratory failure. Chest. 2004;126(2):540-546.

19. Ford ES. Does exercise reduce inflammation? Physical activity and C- 
reactive protein among U.S. adults.

Epidemiology. 2002;13(5):561-568.

20. Yousef AM, Alkhiary W. Role of neutrophil to lymphocyte ratio in prediction of acute exacerbation of chronic obstructive pulmonary disease. Egypt $J$ Chest Dis Tuberc. 2017;66(1):43-48. 